\title{
PWE-026 COMPARISON OF MEDICAL COSTS AMONG PATIENTS USING ADALIMUMAB AND INFLIXIMAB: A RETROSPECTIVE STUDY (COMPAIRS)
}

Introduction Anti-tumour necrosis factor (anti-TNF) medications have never been compared in a direct fashion under real-world circumstances. The purpose of this study was to compare healthcare utilisation and costs using insurance data for patients with Crohn's disease (CD) who newly initiated antiTNF therapy with adalimumab (ADA) or infliximab (IFX).

Methods Patients with $\geq 2$ diagnoses of CD (ICD-9-CM: 555. XX) who initiated ADA or IFX therapy between January 2000 and March 2009 were identified from the Medstat MarketScan database. Patients had to be continuously enrolled at least 6 months before and after anti-TNF initiation. Patients with prior biologic therapy (ie, anti-TNF or natalizumab) or colitis (ICD-9-CM: 556.x) were excluded. ADA and IFX groups were matched 1:1 using a propensity score stratified by age, residence, inpatient visit utilisation and steroid use at baseline. The primary endpoint was 6 -month direct cost of healthcare. The secondary endpoints compared healthcare utilisation between groups.

Results After propensity matching, demographic, clinical and healthcare utilisation characteristics were similar between the ADA $(n=623)$ and IFX $(n=623)$ groups at baseline. During the 6-month interval following anti-TNF initiation, healthcare costs were significantly lower for ADA compared with IFX therapy (table 1).

Both all-cause and CD-related hospitalisation decreased for both ADA and IFX groups (not shown). Emergency department (ED) use and hospitalisation in the 6-month follow-up period were similar between groups.

Conclusion In this real-world analysis of patients with CD who newly initiated with ADA or IFX, ADA-treated patients had significantly lower healthcare costs. Hospitalisation and ED utilisation were similar between groups.

Competing interests D. Sussman: None Declared, N. Kubiliun: None Declared, J. Chao Shareholder with: Abbott, Employee of: Abbott, P. Mulani Shareholder with: Abbott, Employee of: Abbott, C. Gillis Shareholder with: Abbott, Employee of: Abbott, M. Yang Shareholder with: Abbott, Employee of: Abbott, M. Lu Employee of: Analysis Group; under contract with Abb., M. Abreu Consultant for: Abbott, Amgen, Elan, Opson, Prometheus, Salix, UCB

Keywords Crohn's disease, healthcare costs, TNF-alpha inhibitors.

doi:10.1136/gut.2011.239301.289

D A Sussman, ${ }^{1}$ N Kubiliun, ${ }^{1} \mathrm{~J}$ Chao, ${ }^{2, *}$ P M Mulani, ${ }^{2}$ C A Gillis, ${ }^{2}$ M Yang, ${ }^{2}$ M Lu, ${ }^{3}$ M T Abreu' 1 University of Miami, Miami, FL, USA; ${ }^{2}$ Abbott Laboratories, Abbott Park, IL, USA; ${ }^{3}$ Analysis Group, Inc., Boston, MA, USA

Table 1 PWE-026 Mean HealthCare Costs (Per Person) During the 6 Months After Initiating Anti-TNF Therapy (US \$)

\begin{tabular}{lllll}
\hline & $\begin{array}{l}\text { All-Cause, ADA } \\
(\mathbf{n = 6 2 3})\end{array}$ & $\begin{array}{l}\text { All-Cause, IFX } \\
(\mathbf{n}=\mathbf{6 2 3})\end{array}$ & $\begin{array}{l}\text { CD-Related } \\
(\mathbf{n}=\mathbf{6 2 3})\end{array}$ & $\begin{array}{l}\text { CD-Related } \\
\text { (n=623) }\end{array}$ \\
\hline Anti-TNF drug costs & - & - & 10709 & 12401 \\
Other prescription drug costs & $1334^{\mathrm{b}}$ & 1639 & 546 & 857 \\
Total medical service-related costs & $6842^{\mathrm{b}}$ & 10316 & $5199^{\mathrm{b}}$ & 9059 \\
Total healthcare costs & $\mathbf{1 8 ~ 8 8 5}^{\mathrm{b}}$ & $\mathbf{2 4} \mathbf{3 5 5}$ & $\mathbf{1 6 4 5 4 ^ { \mathrm { b } }}$ & $\mathbf{2 2} \mathbf{3 1 6}$ \\
(costs excluding anti-TNF agents) & $\left(8176^{\mathrm{b}}\right)$ & $(11955)$ & $\left(5745^{\mathrm{b}}\right)$ & $(9916)$ \\
\hline
\end{tabular}

aBased on diagnosis of $\mathrm{CD}$ or related comorbidities (eg, malnutrition, anaemia, abdominal symptoms, etc).

${ }^{b} p<0.0005$ vs IFX.

'Medical costs other than hospitalisation and outpatient visits are not shown. 